\title{
Análisis de los fenómenos de estallido y exfoliación en piezas prefabricadas pretensadas no convencionales
}

\section{Study of spalling and bursting forces in precast prestressed concrete elements with no conventional geometry}

\author{
J. Rueda Contreras ${ }^{(*)}, \underline{\text { D. Fernández Montes }}{ }^{(* *)}$, E. González Valle ${ }^{(* * *)}$
}

\section{RESUMEN}

El presente artículo profundiza en los fenómenos de estallido y exfoliación producidos en elementos pretesos, centrándose en piezas sin armadura transversal y con configuración no convencional. Partiendo de casos de patología documentados, se analizan ambos fenómenos. Se estudian los medios disponibles de predicción y evaluación de las tensiones asociadas, analizando la validez de su uso. Para este último fin, se realiza un estudio mediante Elementos Finitos, localizándose parámetros geométricos y mecánicos relevantes. Finalmente, se concluye con la exposición de puntos fundamentales para el correcto tratamiento del estallido y la exfoliación en este tipo de piezas, pero que no han sido suficientemente desarrollados en la bibliografía o en las normas y códigos aplicables.

Palabras clave: Hormigón pretensado, transferencia del pretensado, prefabricado, adherencia, estallido, exfoliación, pretesado.

\section{ABSTRACT}

Spalling and bursting forces in prestressed concrete members are addressed in this current Manuscript. Specifically, it is focused on structural members with no conventional geometry and without transverse reinforcement. Starting out by some informed forensic cases, both forces and related tensile stresses are being analyzed. The approaches for the prediction and evaluation of these stresses are also analyzed and the validity is properly verified. For this purpose, a Finite Element Analysis is made, underlining some geometrical and mechanical parameters. Finally, technical aspects (which could be essential for the structural analysis of the spalling and bursting forces in these kind of specimens) that have not been carried out in deep in the referenced technical documents or in the current codes or specifications are highlighted.

Keywords: Prestressed concrete, built-up of prestress, precast, bond, bursting, spalling, prestressed.

(*) Dr. Ingeniero de Caminos, Canales y Puertos; OHL Industrial; Madrid, España.

(**) Dr. Ingeniero de Caminos, Canales y Puertos; Profesor de la ETS Ingeniería Civil de la UPM; Madrid, España.

(***) Dr. Ingeniero de Caminos, Canales y Puertos; ExProfesor de la ETS Caminos, Canales y Puertos de la UPM; Madrid, España.

Persona de contacto/Corresponding author: david.fernandez.montes@upm.es (D. Fernández Montes).

ORCID: https://orcid.org/o0oo-0002-8310-757X (J. Rueda Contreras); https://orcid.org/oooo-0001-5900-6704

(D. Fernández Montes); https://orcid.org/oooo-0o02-2759-5097 (E. González Valle)

Cómo citar este artículo/Citation: Rueda Contreras, J.; Fernández Montes, D.; González Valle, E. (2019). Análisis de los fenomenos de estallido y exfoliación en piezas prefabricadas pretensadas no convencionales. Informes de la Construcción, 71(555): e297. https://doi. org/10.3989/ic.64053

Copyright: (C) 2019 CSIC. Este es un artículo de acceso abierto distribuido bajo los términos de la licencia de uso y distribución Creative Commons Reconocimiento 4.0 Internacional (CC BY 4.0). 


\section{TIPOLOGÍA OBJETO DE ESTUDIO}

El empleo de elementos prefabricados en edificación presenta una constante evolución (1) (2). A principio del siglo actual se desarrolló una nueva tipología de piezas prefabricadas para forjados unidireccionales (3) (4). Desde su desarrollo, ha sufrido casos documentados de patología. En varios de ellos (5) (6) (7), el colapso de las piezas se produjo con carácter frágil.

La tipología referida consiste en una pieza prefabricada con armadura pretesa cuya sección transversal consiste básicamente en una losa inferior, en la que se aloja la cuantía principal de acero pretensado, y una serie de nervios, generalmente cuatro, asociados por parejas. No presentan armadura transversal. Sobre la losa inferior se aloja el elemento de aligeramiento y, una vez puesta en obra, se hormigona in situ la losa superior y el espacio entre nervios adyacente, configurándose el forjado de modo definitivo, tal y como se muestra en la Figura 1.

Los valores frecuentes de las piezas de dicho forjado se enumeran a continuación:

- Canto $(H): 15 \mathrm{~cm}-25 \mathrm{~cm}$.

- Espesor de la losa inferior $\left(e_{t}\right): 3,9 \mathrm{~cm}-5,2 \mathrm{~cm}$ (normal) o $6,5 \mathrm{~cm}$ (protección frente al fuego).

- Espesor mínimo de nervio $(a): 4-6,4 \mathrm{~cm}$.

- Relación altura del centro de gravedad / canto $\left(y_{g} / H\right)$ : $0,31-0,34$.

- Relación entre el espesor de la losa inferior y la posición del pretensado inferior: 0,46 - o,56 (sin contar los casos diseñados frente a incendios).

- Fuerza de pretensado total en el momento de la transferencia $\left(N_{p t}\right): 391-816 \mathrm{kN}$.

- Relación excentricidad de $N_{p t} /$ Canto $\left(e_{p} / H\right)$ : 0,05 - o,12.

- Fuerza de pretensado inferior en el momento de la transferencia $\left(N_{p t, i}\right): 325-727 \mathrm{kN}$.

- Relación excentricidad de $N_{p t, i} /$ Canto $\left(e_{p i} / H\right): 0,08$ - o,21.

- Fuerza de pretensado superior en el momento de la transferencia $\left(N_{p t, s}\right): 55-100 \mathrm{kN}$.

- Relación excentricidad de $N_{p t, s} /$ Canto $\left(e_{p s} / H\right): 0,37-0,58$.

Las piezas se producen habitualmente con un ancho de $120 \mathrm{~cm}$, y el espesor de la losa inferior se encuentra comprendido entre 4 y 6,5 cm. Las soluciones con espesores entre 6 y
$6,5 \mathrm{~cm}$ tienen como objetivo una mayor resistencia frente al fuego.

El canto total de las piezas es de $20 \mathrm{~cm}$ como media, descendiendo en ocasiones hasta $12 \mathrm{~cm}$, y llegando a ascender hasta alcanzar los $26 \mathrm{~cm}$. En cuanto al espesor mínimo de los nervios, este varía entre 3 y $6,5 \mathrm{~cm}$.

La cuantía de armadura longitudinal pretesa varía, para una misma geometría de la sección transversal, en función de la capacidad resistente requerida. Está compuesta por alambres de diámetros de 4 o $5 \mathrm{~mm}$ y, ocasionalmente, cordones. La mayor parte se aloja en la losa inferior, en un mismo nivel o capa, y también se dispone un tendón en la cabeza de cada nervio. Dentro de la losa inferior, ocasionalmente se distribuyen uniformemente los tendones a lo largo del ancho de la pieza, mientras en otras se agrupan en las proximidades de los nervios. Frecuentemente se alojan distintos niveles de tendones en el plano medio de los nervios.

Las diferentes casas comerciales producen piezas con varias geometrías de sección transversal, para cada una de las cuales existen diversas configuraciones de armado. Al conjunto de piezas con una misma geometría de la sección transversal se las denominará en adelante familia. Asimismo, en la Figura 1 se indican los valores frecuentes de diversos parámetros de la sección transversal, obtenidos de una base de datos de 131 placas comerciales, correspondientes a 20 familias y 7 prefabricadores diferentes. Las diferentes características de los elementos que componen dicha base son definidos en (7).

\section{DESCRIPCIÓN DE CASOS DE PATOLOGÍA DOCUMENTADOS}

Un primer estudio analizó el colapso de varias piezas en una obra en fase de ejecución.

El fallo ocurrió de madrugada, y cuando sucedió las cargas actuantes consistían únicamente en el peso propio de las placas y del hormigón in situ de la losa superior y los nervios. En tres placas contiguas se produjo el colapso por rotura del encuentro entre los nervios y la losa inferior, de modo frágil. La losa inferior llegó a desprenderse cayendo sobre el piso inferior, o quedó colgando de los alambres, tal y como muestra la Figura 2a).

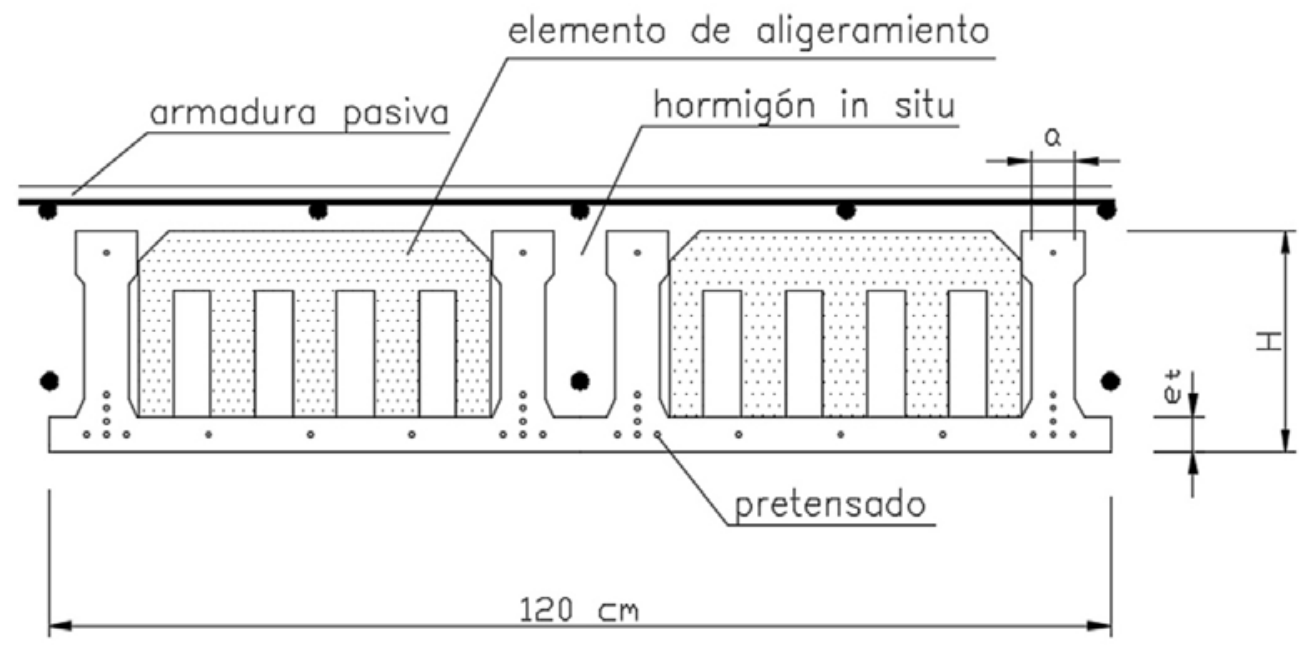

Figura 1. Trozo de la costa que correspondía al reino de Granada. 


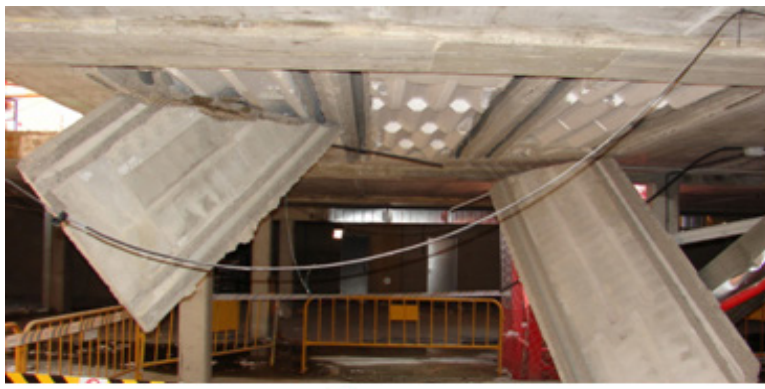

a)

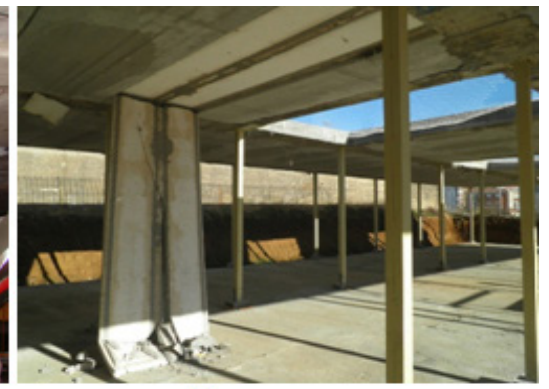

b)

Figura 2. a) Colapso de tres placas en obra en curso. b) Colapso de una placa en obra cerrada.

De acuerdo con las especificaciones, las placas debían ser de $25 \mathrm{~cm}$ de canto, y se disponían 18 alambres Y1770C de $5 \mathrm{~mm}$ de diámetro en la losa inferior, y 4 de diámetro $4 \mathrm{~mm}$ en la cabeza de los nervios. La resistencia del hormigón a 28 días era de $40 \mathrm{MPa}$.

Durante la inspección de la zona afectada, no se apreciaron anomalías relevantes en la losa superior de los forjados, ni diferencias geométricas entre las placas y sus especificaciones. En cuanto a la armadura activa, si bien no coincidía con la descrita en las fichas de características, la cuantía dispuesta era equivalente.

En el transcurso de la inspección, se comprobó que en una obra próxima se había producido el mismo modo de fallo y se localizaron idénticas piezas acopiadas en las que se apreciaban fisuras en la zona de encuentro entre los nervios y la losa inferior.

Consecuentemente, se visitó la planta de prefabricación dónde se habían producido las placas dañadas. Allí se observó que se fabricaban en pista mediante hormigonado continuo con ponedora, cortándose las piezas a la longitud requerida tras pasar al menos dos días. También se comunicó que se respetaba la cuantía de armadura pretensada de las especificaciones, pero no el diámetro de la misma o su distribución transversal.

Se comprobó que, entre las piezas acopiadas en la planta, especialmente en las de mayor cuantía de pretensado, aparecían fisuras en la zona de encuentro entre el nervio y la losa inferior, como muestra la Figura 3. Asimismo, se informó que, ocasionalmente, la aceptación de las placas no era aprobada debido a este tipo de fisuración.

El estudio concluyó que la causa más probable de la fisuración y rotura de las piezas era la alta concentración de tensiones de tracción en la zona del encuentro entre los nervios y la losa inferior. Se consideró la transferencia del pretensa-

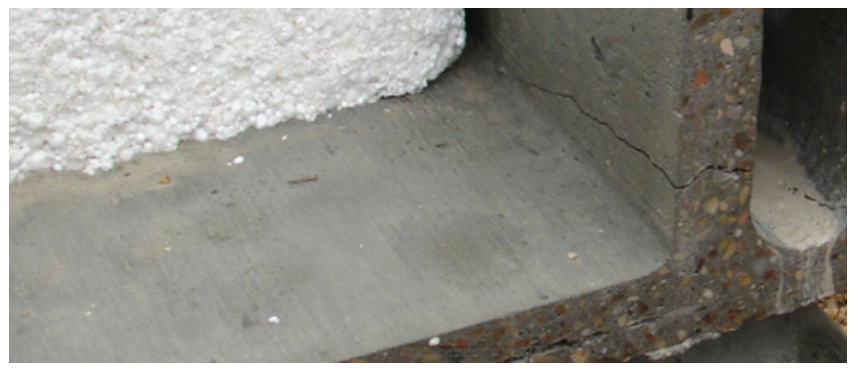

Figura 3. Placa acopiada en la planta con fisura en un nervio. do como el origen más probable de la aparición de tensiones tangenciales excesivas, concluyéndose que los márgenes de seguridad existentes frente a estas tensiones en el momento de la transferencia eran insuficientes.

Posteriormente se llevó a cabo un segundo estudio. En este caso se trató de dos piezas, no contiguas, colocadas en una obra que llevaba varios meses parada. En una visita periódica, se descubrió el colapso de las placas, como se muestra en la Figura 2b).

Las cargas actuantes eran únicamente el peso propio de la placa y el del hormigón in situ para la terminación del forjado. Por otro lado, si bien se trataba de la misma tipología y del mismo modo de fallo, en esta ocasión se trataba de otro diseño y otro fabricante y por tanto la geometría de la sección transversal y la configuración del pretensado eran diferentes.

En este caso se trataba de piezas de $13 \mathrm{~cm}$ de canto, armadas con 8 alambres Y1860 de diámetro $5 \mathrm{~mm}$ en la losa inferior, y uno en cada cabeza de nervio.

Se concluyó que las causas de la fisuración y rotura de las piezas coincidían con las indicadas para el caso anterior, si bien el arranque de la rotura parecía localizarse en un nivel intermedio del nervio.

\section{DESCRIPCIÓN DE LOS FENÓMENOS DE ESTALLIDO Y EXFOLIACIÓN}

\subsection{Estado tensional de la pieza}

En la zona de transferencia del pretensado, la tensión aplicada a la armadura pretesa se transmite a la sección de hormigón, pasando de encontrarse en un estado libre de tensiones en el extremo, a un estado tensional de equilibrio con la fuerza de pretensado transferida. La distancia comprendida entre el extremo de la pieza y la sección en que se ha transferido la totalidad de la fuerza de pretensado introducida en el tendón se denomina longitud de transmisión. Las tensiones en la zona de transferencia no seguirán, de forma general, una distribución lineal. Estas condiciones especiales hacen que aparezcan tensiones transversales de tracción en el hormigón.

Por tanto, se trata de una zona de discontinuidad o región $\mathrm{D}$, que suele estudiarse mediante el método de bielas y tirantes (8). Su empleo corresponde a un enfoque en Estado Límite Último, en el que el hormigón está fisurado y las tensiones de tracción son resistidas por las armaduras. Siendo así no es un enfoque adecuado para elementos pretensados sin armadura transversal (9) (10) (11). 


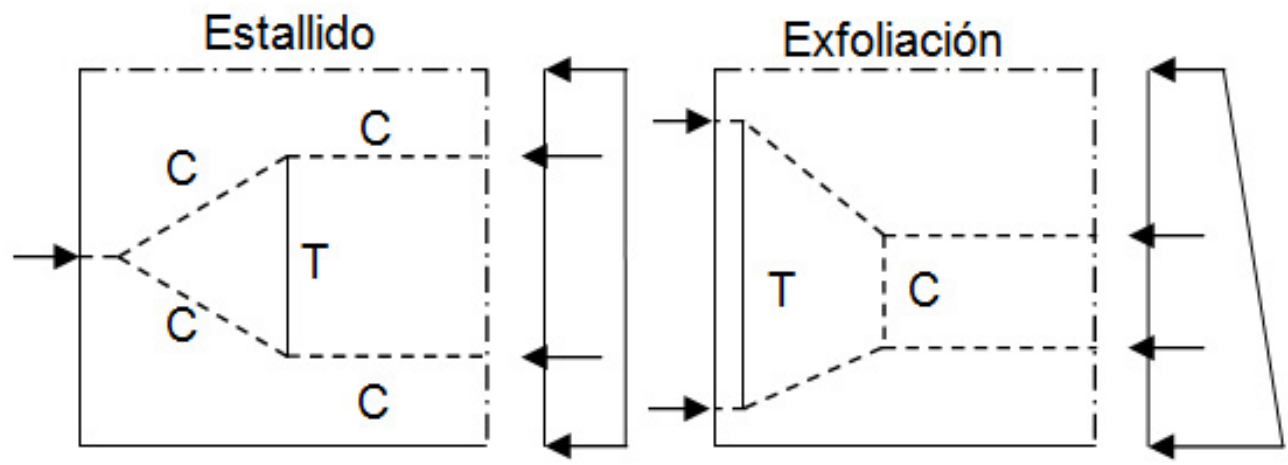

Figura 4. Esquema simplificado de tensiones en la zona de transferencia.

Las tracciones provocadas por la transmisión del pretensado son de tres tipos, hendimiento (splitting), exfoliación (spalling) y estallido (bursting) (11) (12). El hendimiento se produce por efecto directo de la adherencia entre el acero pretensado y el hormigón. Origina una fisura en la interfaz entre el tendón y el hormigón, que avanza hacia la superficie más cercana, ya sea el exterior del elemento, ya sea otro tendón. Las tensiones de exfoliación y estallido se deben a la configuración geométrica del conjunto hormigón - armadura activa y a la distribución de la fuerza de pretensado a lo largo de la sección transversal y la zona de transferencia. Las primeras provocan tracciones próximas al extremo del elemento, debido a la asimetría del punto de aplicación de la carga. Las últimas las producen en una zona interior y aproximadamente en la línea de acción de la carga, fruto de la dispersión de la fuerza del pretensado, y por tanto independientemente de la excentricidad existente. En la Figura 4 puede observarse una representación esquemática de estas tensiones.

Tanto las tensiones de exfoliación como las de estallido provocan la aparición de fisuras, en general horizontales, que se originan en las zonas de actuación de las tensiones, y que evolucionan durante los días y semanas posteriores a la transferencia del pretensado (12). Además, es importante destacar que la tensión de exfoliación depende del punto de aplicación de la carga, y de la posición de la resultante de las fuerzas actuantes. Así, si se dispone pretensado superior e inferior, el estado tensional será la combinación de los dos estados individuales.

\subsection{Descripción de los métodos disponibles}

El problema de la presencia de tracciones en la zona de transferencia ha sido tratado con frecuencia en la bibliografía, siendo varios los métodos disponibles para su evaluación.

Los primeros datan de los años 50 y 60 del siglo veinte. Basado en el análisis elástico bidimensional de piezas con armadura postesa de sección rectangular con pretensado centrado, Guyon desarrolló la analogía del prisma simétrico (13) para el cálculo de las tensiones de estallido,la cual sigue en vigor hoy día (14), generalizada tanto para diferentes secciones y distribuciones de pretensado, como para elementos con armadura pretesa (12) (15).

En lo que se refiere a las tensiones de exfoliación, Gergely y Sozen desarrollaron en los años 60 un método de cálculo (16) (17), denominado por el Código Modelo 9o (CM9o) analogía del prisma equivalente. Básicamente, consiste en el plantea- miento del equilibrio del cuerpo libre resultante tras la aparición de la primera fisura, y tiene en cuenta tanto la geometría de la sección transversal, como la posición del conjunto de la armadura pretensada. El método permite predecir la posición de la primera fisura, y la fuerza de tracción correspondiente, y se generaliza para secciones con armadura pretesa. Como en el caso del método de Guyon, la analogía del prisma equivalente sigue en vigor a día de hoy (18). La aplicación de ambas analogías para piezas con armadurapretesasin armadura transversal puede encontrarse en el Código Modelo 2010 (CM10) (14) y el CM9o (11).

Correspondientes también a los años 60, las investigaciones de Marshall y Mattock (19), y de Arthur y Ganguli (20), obtuvieron expresiones que permitían calcular la cuantía de estribos necesaria para resistir las tracciones producidas en la zona de transferencia. En general, estos trabajos señalan que las fisuras aparecen entorno al centro de gravedad de las piezas o en las zonas de unión entre ala y alma, y se centran en el cálculo de la armadura transversal necesaria para controlar dicha fisuración. Si bien este enfoque se mantiene de actualidad y en constante actualización (15) (18) (21), en general no es aplicable a las piezas prefabricadas para forjados, ya que tanto los procesos constructivos como las ajustadas seciones transversales con frecuencia no permiten la disposición de armadura transversal.

Durante los años 8o, Den Uijl trabajó en el campo de las losas alveolares (12). Adaptó la analogía del prisma simétrico a las condiciones específicas de estas, y desarrolló la siguiente expresión que permite obtener el valor máximo de la tensión de estallido. La expresión se incluye en (22) y, como ábaco, en el CM9o y el CM10 de forma generalizada para secciones con armadura pretesa:

$$
\sigma_{s p}=\frac{2\left(0,02+4 \alpha^{2,3}\right) \cdot(\alpha+1 / 6)}{(0,1+0,5 \alpha)\left[1+1,5\left(L_{t} / e\right)^{1,5} \cdot(\alpha+1 / 6)^{1,5}\right]} \cdot \frac{F_{p e f}}{t \cdot e}, \operatorname{con} \alpha=\frac{e-k}{H}
$$

$\sigma_{s p}$ Tensión de exfoliación.

$e^{s p}$ Excentricidad del pretensado.

$k$ Distancia comprendida entre el centro de gravedad de la pieza y el extremo del núcleo central más próximo al tendón.

$L_{t}$ Longitud de transmisión.

$H$ Canto de la pieza.

$t$ Ancho del alma.

$F_{p e f}$ Fuerza de pretensado eficaz. 
La Norma EN 1168de placas alveolares (23) incluye una expresión que modifica la anterior, si bien ofrece resultados similares:

$$
\sigma_{s p}=\frac{15 \cdot \alpha^{2,3}+0,07}{1+\left(L_{t} / e\right)^{1,5} \cdot(1,3 \cdot \alpha+0,1)} \cdot \frac{F_{p e f}}{t \cdot e}, \operatorname{con} \alpha=\frac{e-k}{H}
$$

Mediante estas expresiones se obtendría el valor de la tensión máxima de exfoliación, que debería compararse con el valor de resistencia a tracción del hormigón en el momento del tesado.

Por último, cabe destacar que los trabajos de Eriksson (21) incluyenexpresiones obtenidas para el cálculo de la tracción en el hormigón por exfoliación, bien la fuerza de tracción $T_{s p}$, bien la tensión $\sigma_{s p}$.

$$
\begin{gathered}
\sigma_{s p}=\frac{F_{p i}}{A}\left(0,1206 \cdot \frac{e^{2}}{H \cdot \varnothing}-0,0256\right) \geq 0 \\
\frac{T_{s p}}{F_{p i}}=0,02 \cdot \frac{e^{2}}{H \cdot \varnothing}-0,01 \geq 0
\end{gathered}
$$

A Área bruta de la sección.

f Diámetro del tendón.

$F_{p i}$ Fuerza de pretensado al tesar.

La fuerza de tracción debe considerarse actuando en una distancia medida desde el extremo de la pieza de $H / 12$.

Tanto los trabajos de Den Uijl como de Eriksson incluyeron el análisis de las secciones mediante elementos finitos lineales bidimensionales, y permiten evaluar las tensiones de tracción por exfoliación producidas en piezas sin armadura transversal.

\section{INVESTIGACIÓN REALIZADA}

Los estudios reseñados anteriormente evidencian un posible problema no resuelto en la tipología de piezas descritas. Ante esta situación, se llevó a cabo una investigación en la Universidad Politécnica de Madrid. Inicialmente, se aplicaron los métodos de cálculo indicados. El objetivo era comprobar si arrojaban resultados coherentes entre sí, y si dichos resultados justifican la aparición de daños. A continuación, se procedió al análisis de la tipología mediante modelos de elementos finitos de piezas teóricas, con el objeto de profundizar en el estudio considerando diversos factores geométricos y mecánicos. Finalmente, se analizaron los focos de incertidumbre que, en este caso, acentúan el riesgo de fallo de las piezas.

\subsection{Aplicación de los métodos disponibles a la base de datos de piezas reales}

Se analizó el estado tensional en la zona de transferencia de los elementos que componen la base de datos ya descrita. Con este fin, se aplicaron los métodos de cálculo señalados anteriormente: analogía del prisma simétrico, analogía del prisma equivalente, ecuación de Den Uijl, ecuación de EN 1168, y ecuaciones de Eriksson.
Con frecuencia, la base de datos carece de información relevante para el análisis de las tensiones en la zona de transferencia, como por ejemplo la edad de transferencia de pretensado. Para solventar este problema, se adoptaron valores habituales de elementos reales para todos los elementos: resistencia característica del hormigón a 28 días $\left(f_{c k}\right)$ de 45 $\mathrm{MPa}$, transferencia efectuada al alcanzar el hormigón una resistencia media a compresión $\left(f_{c m, t}\right)$ de $30 \mathrm{MPa}$ y resistencia característica a tracción en el momento de la transferencia $\left(f_{\text {ctk,t }}\right)$ de 1,54 MPa. Las características del acero de pretensar varían de unos elementos a otros, consistiendo mayoritariamente en alambres de diámetros $4 \mathrm{~mm}$ o $5 \mathrm{~mm}$. En la referencia (7) pueden encontrarse más información de los elementos que componen la base de datos. La longitud de transferencia del pretensado se calculó de acuerdo con CM9o.

Se calcularon las tensiones de estallido en el momento de la transferencia mediante la analogía del prisma simétrico, adoptando diversas hipótesis a la hora de considerar la forma de la sección transversal y la existencia de distintos niveles de pretensado. Las tracciones calculadas superaron el valor de $f_{c t k, t}$ entre el 95\% y el o\% de los casos (6). Los resultados más desfavorables se obtuvieron al emplear la adaptación de la analogía a placas alveolares desarrollada por Den Uijl (12) y considerar el nivel inferior de pretensado. Los más favorables se consiguieron al contemplar el conjunto del pretensado inferior y adoptar como ancho del prisma el correspondiente a la fibra en que se encuentra el acero de pretensar, que usualmente es la losa inferior.

Para la obtención de las tensiones de exfoliación se empleó la analogía del prisma equivalente y las ecuaciones [1] [2] [3] [4]. En el caso de la ecuación [4], la tensión se calculó considerando la fuerza de tracción en una sección de pieza de longitud $H / 12$ y ancho, el mínimo de la pieza.

De estos cinco métodos, sólo mediante la analogía del prisma equivalente se obtiene la distribución de tensiones en función de la altura y para el conjunto del pretensado actuante en la sección. Mediante las ecuaciones [1] a [4] se obtiene el valor de la tensión máxima, pero no su posición. Además, se aplican por separado al pretensado superior e inferior. Así, la tensión máxima del conjunto del pretensado sería la combinación lineal de los máximos obtenidos individualmente para el pretensado inferior y superior. Dado que estos no tienen por qué coincidir en posición, no serán directamente sumables. Sí se puede considerar que la tensión máxima se encontrará en un intervalo que presenta como cota superior la suma de las tensiones máximas individuales, y como cota inferior, la máxima de estas. Matemáticamente:

$$
\sum\left(\sigma_{s p, i} ; \sigma_{s p, s}\right) \geq \sigma_{s p} \geq \operatorname{máx}\left(\sigma_{s p, i} ; \sigma_{s p, s}\right)
$$

\section{Siendo:}

$s_{s p} \quad$ Tensión máxima de exfoliación.

$s_{s p, i}$ Tensión máxima de exfoliación debida a la acción del pretensado inferior.

$s_{s p, s}$ Tensión máxima de exfoliación debida a la acción del pretensado superior.

En la Tabla 1 se indica el porcentaje de casos en que las tensiones de exfoliación obtenidas superaron el valor $f_{c t k, t^{*}}$ 
Los resultados obtenidos de la aplicación de las ecuaciones [1] y [2] son muy similares, con una diferencia media entre sí del $3 \%$, y siempre inferior al 10\%. Por sencillez de la exposición, en adelante se hará referencia únicamente a los resultados de la aplicación de la ecuación [1].

Tabla 1. Porcentaje de casos en que se supera $f_{\text {ctk, } t \text {. Exfoliación. }}$

\begin{tabular}{|l|c|c|c|c|c|}
\hline & $\begin{array}{c}\text { Prisma } \\
\text { equivalente }\end{array}$ & [1] & [2] & [3] & [4] \\
\hline$S_{s p}$ & $26 \%$ & --- & --- & --- & --- \\
\hline$S_{s p . i}$ & --- & $24 \%$ & $23 \%$ & $5 \%$ & $38 \%$ \\
\hline$S_{s p . s}$ & --- & $8 \%$ & $5 \%$ & $17 \%$ & $53 \%$ \\
\hline
\end{tabular}

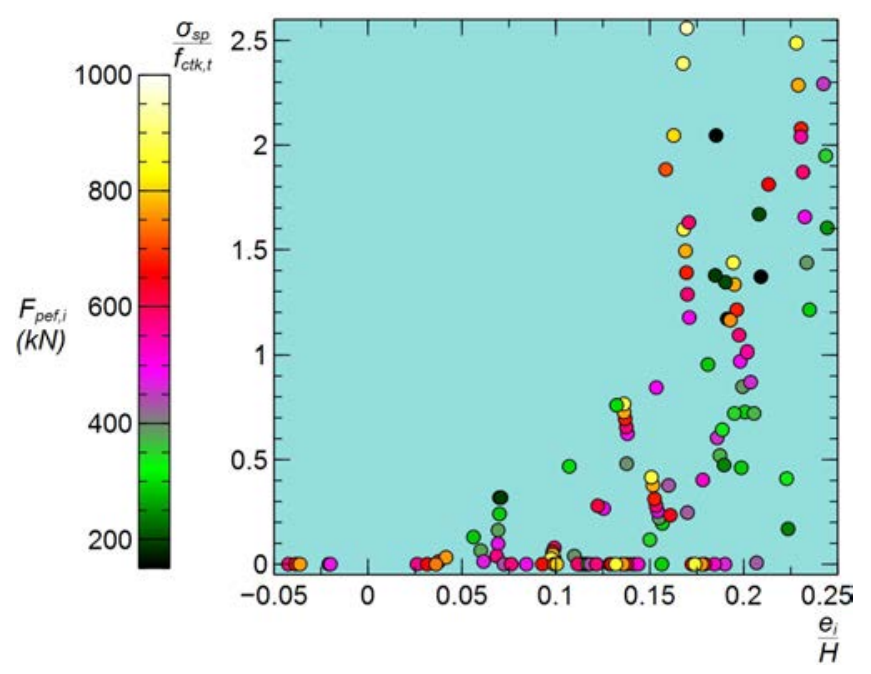

Figura 5. Resultados obtenidos. Analogía del prisma equivalente.
Los resultados del cálculo de las tensiones de tracción por exfoliación para cada una de las piezas de la base de datos se muestran en las gráficas siguientes (Figura 5 y Figura 6), en función del valor de la tensión de exfoliación relativa a $f_{c t k, t}, \mathrm{y}$ de la excentricidad relativa del pretensado inferior, $e_{i}$, o superior, $e_{s}$, al canto, $H$.

En algunos casos de la Figura 6, el valor de la tensión obtenida es cero. Esto se debe a que, para que las ecuaciones empleadas sean aplicables, la excentricidad del pretensado debe superar un valor mínimo. Físicamente, esta condición representa el hecho de que cuando la excentricidad es muy pequeña, el estado de tensión producido es próximo a la compresión centrada. Este fenómeno se recoge en las ecuaciones [1] y [2] mediante el factor $(e-k)$, y también puede encontrarse en los resultados de la aplicación de la analogía del prisma equivalente, Figura 5.

Con el objeto de establecer una comparación, se emplearon las ecuaciones [1] a [4] considerando la resultante del pretensado total. Con ninguno de los procedimientos se superó el valor de $f_{\text {ctk,t }}$ en más de un $5 \%$ de los casos, lo que contrasta con los resultados recogidos en la Tabla 1 . Se confirma así la necesidad de considerar el estado tensional total como combinación de los estados individuales.

Finalmente, en la Figura 7 se representa, para una de las familias que componen la base de datos, el intervalo definido por la ecuación [5] en el que se encontraría la tensión de exfoliación total. En el gráfico se ha añadido, a modo de comparación, el valor de tensión obtenido empleando la resultante total del pretensado en los cálculos.
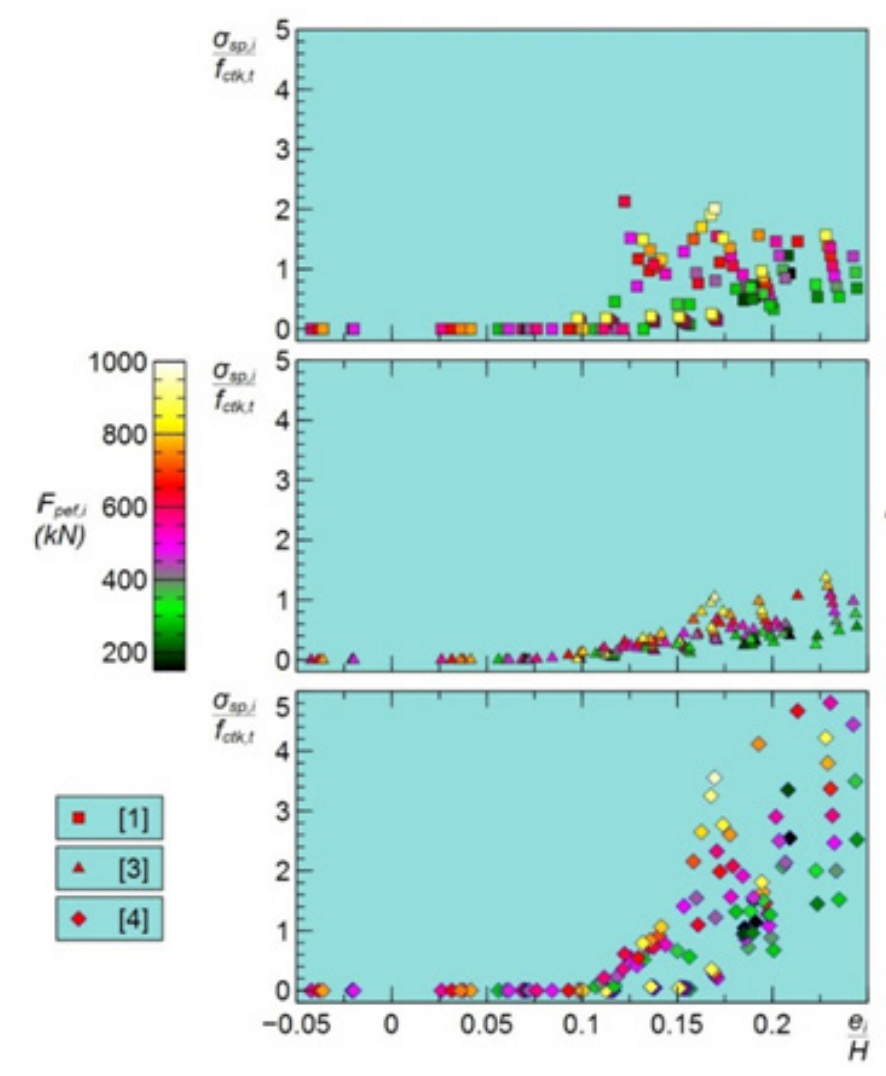

a)

Figura 6. Resultados obtenidos. Ecuaciones [1], [4] y [3], a) pretensado inferior b) pretensado superior.

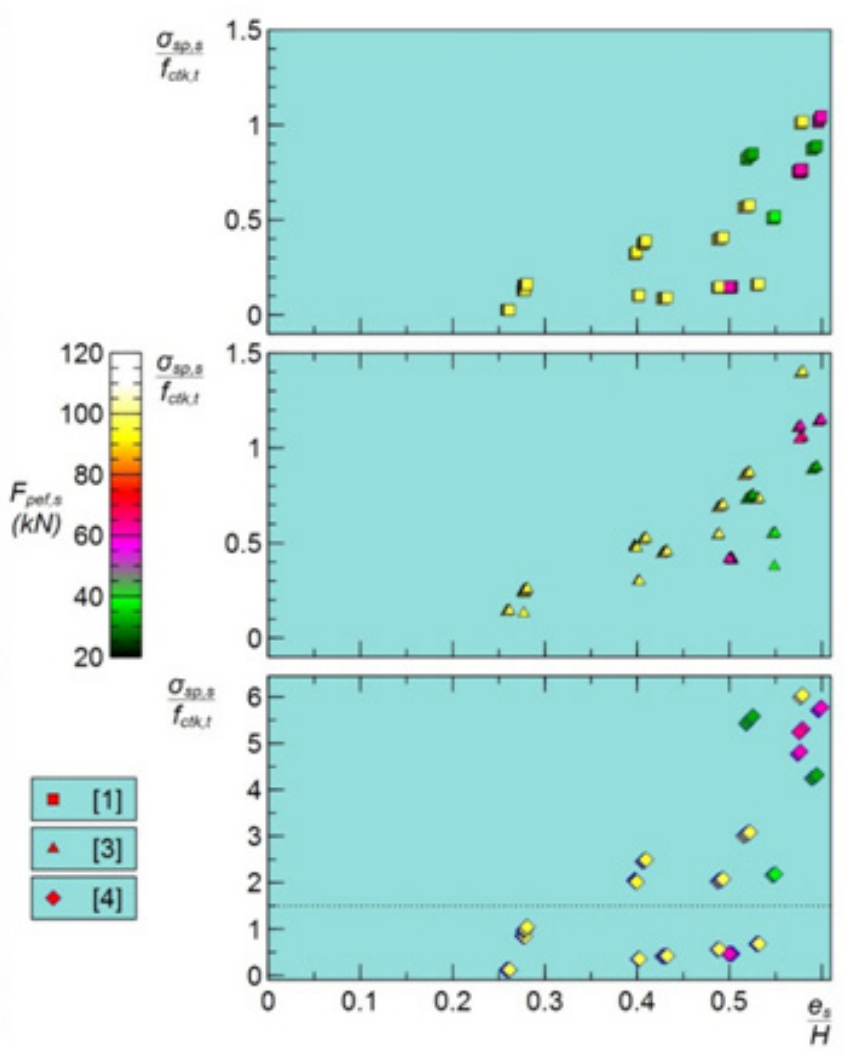

b) 

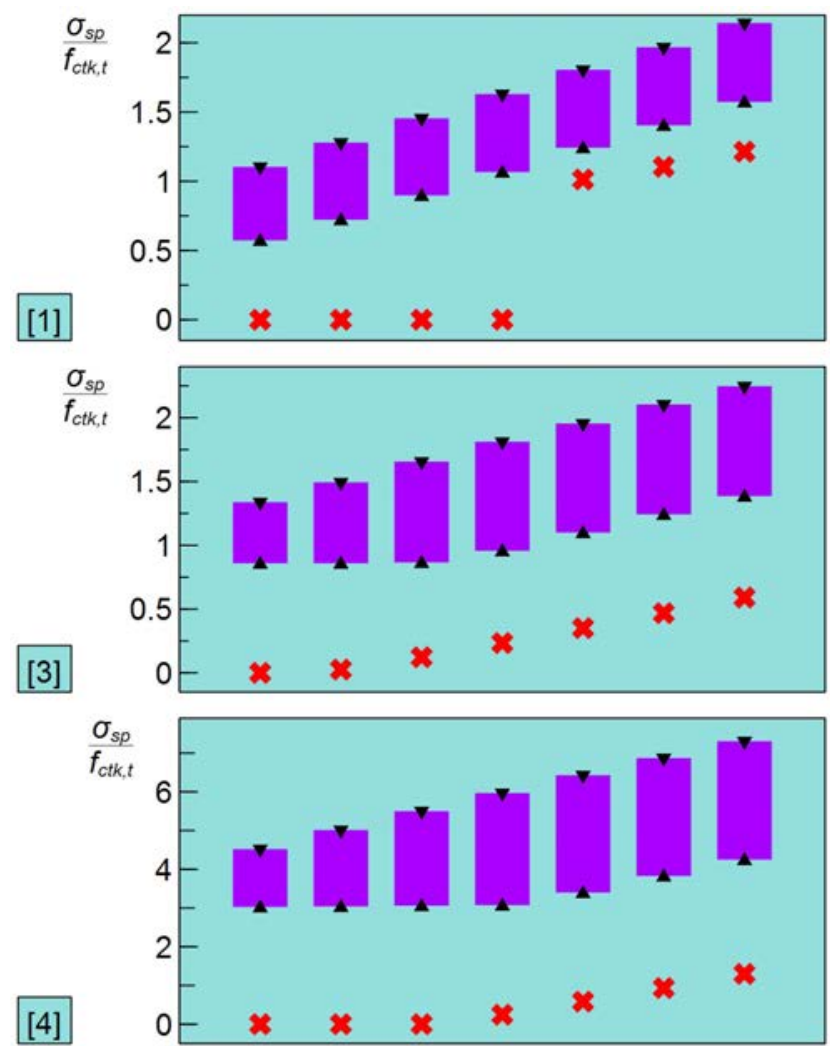

\section{$\Delta$ Cota inferior $\vee$ Cota superior $* \sigma_{\text {sp, }}$}

Figura 7. Intervalo de tensiones de exfoliación para la familia B-e de la base de datos (7).

\subsection{Análisis de piezas teóricas de la tipología mediante elementos finitos}

Con el objeto de complementar los resultados señalados, se llevó a cabo un estudio del estado tensional en la zona de transferencia de la tipología tratada mediante un análisis lineal tridimensional por el método de elementos finitos (7) de piezas teóricas. Para ello, se modelizaron 84 piezas, en función de las variables siguientes:

- Canto: $15 \mathrm{~cm}, 20 \mathrm{~cm}$ y $25 \mathrm{~cm}$.

- Espesor de la losa inferior: $5 \mathrm{~cm}$ y 6,5 cm.
- Ancho mínimo de nervios: $4 \mathrm{~cm}$ y $5 \mathrm{~cm}$.

- Cuantía de pretensado: Se consideran tres porcentajes respecto a la cuantía que provoca la máxima compresión admisible, $100 \%$, $75 \%$ y $50 \%$.

- Distribución del pretensado: Dos distribuciones, una con el 70\% del pretensado inferior concentrado bajo los nervios, y otra con sólo el 50\%. El resto se distribuye uniformemente a lo largo de la losa inferior.

- Dos leyes de adherencia, lineal y uniforme.

- Longitud de transmisión según CM9o y ACI 318 (24).

En las piezas modelizadas no se dispone armadura pretensada en el interior de los nervios, con la excepción de un alambre en cabeza. Se dimensiona el pretensado inferior de modo que no aparezcan tracciones en la sección.

Se empleó el programa comercial ANSYS (25), con elementos tipo SOLID186 (Figura 8). Las fuerzas de pretensado se introdujeron como acciones en los nodos, coincidiendo con la posición de los tendones, a lo largo de la zona de transferencia.

En cada uno de los modelos se obtuvo, mediante el análisis por elementos finitos, la máxima tensión de tracción en la zona de encuentro entre la losa inferior y los nervios, $S 1_{\max }$, a lo largo de la longitud de transmisión. Los resultados obtenidos se compararon con los resultados de la aplicación de los métodos de cálculo descritos, sin encontrarse una correlación admisible con ninguno de ellos (Figura 9).

Sin embargo, el análisis de dichos resultados sirvió para localizar varios factores que modifican conjuntamente el valor de la tensión máxima de tracción por exfoliación (7). Estos modificadores son los siguientes, para el caso de adherencia uniforme entre la armadura pretesa y el hormigón:

$$
\begin{aligned}
& \cdot\left(\frac{F_{N}}{F_{p e f, i}}\right)^{0,6} \\
& \text { - }\left(\frac{a}{a_{t}}\right)^{-0,5} \\
& \cdot\left(1+1,4 \frac{F_{p e f, s}}{F_{p e f, i}}\right)^{2}
\end{aligned}
$$

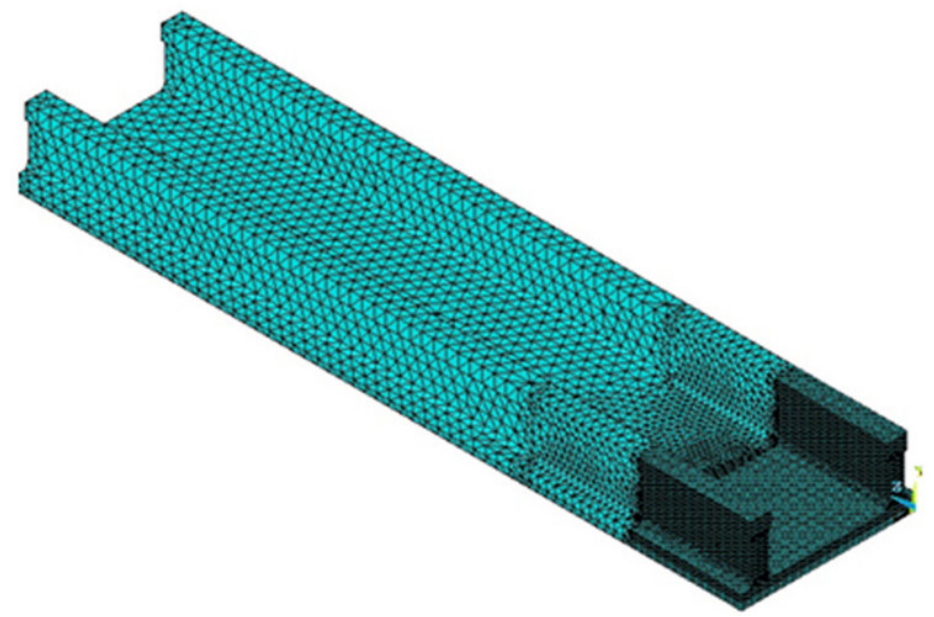

$$
\begin{aligned}
& \text { Ancho de la losa inferior }=120 \mathrm{~cm} \text {. } \\
& \text { Diámetro de alambres }=5 \mathrm{~mm} \text {. } \\
& \text { Designación: Y1860C. } \\
& \text { Tensión de tesado }=1395 \mathrm{~N} / \mathrm{mm}^{2} . \\
& f_{c k}=45 \mathrm{MPa} \text {. } \\
& f_{c m, t}=30,63 \mathrm{MPa} \text {. } \\
& f_{c t, m}=2,19 \mathrm{MPa} \text {. } \\
& E_{c m} \text { (módulo de elasticidad) }=27085 \mathrm{~N} / \mathrm{mm}^{2} \text {. }
\end{aligned}
$$

Figura 8. Vista del modelo de elementos finitos con elementos SOLID186. 


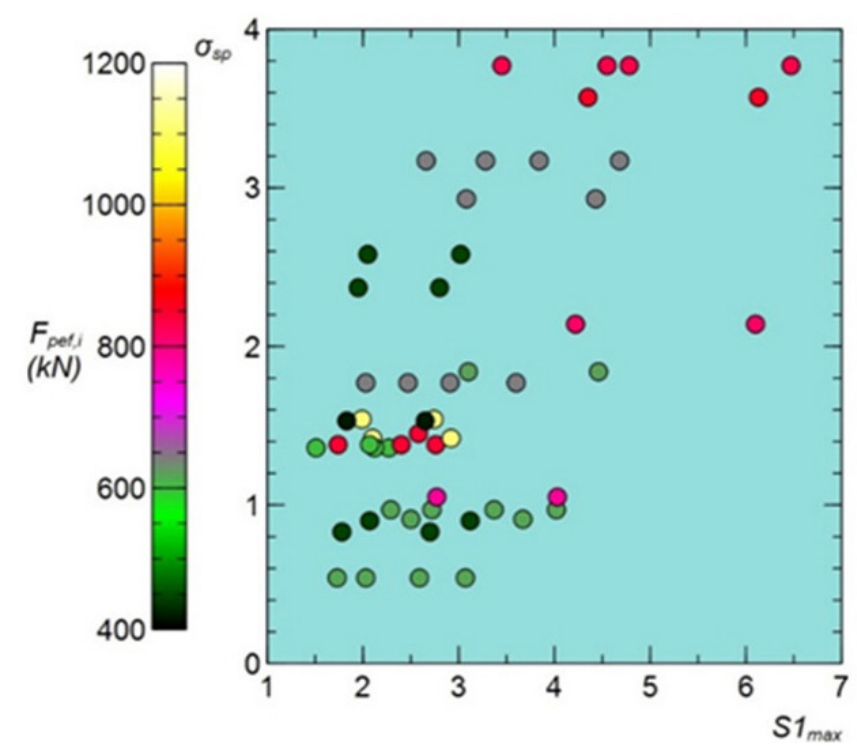

Figura 9. Comparación de los resultados de análisis por elementos finitos y aplicando la analogía del prisma equivalente.

Siendo:

$F_{p e f, i}$ Fuerza de pretensado eficaz inferior.

$F_{N}^{p e f, i}$ Fuerza de pretensado eficaz inferior ubicada bajo los nervios.

$F_{p e f, s}$ Fuerza de pretensado eficaz superior.

$a^{a}$ Ancho mínimo del nervio.

$a_{t} \quad$ Ancho total de la losa inferior.

En la bibliografía consultada no se han encontrado referencias a las relaciones geométricas y mecánicas que representan los factores modificadores señalados.

\subsection{Resultados del estudio}

En definitiva, se observa una gran disparidad de resultados en función del método empleado para calcular las tensiones de tracción en las piezas objeto de estudio. Entre los principales motivos se apuntan los siguientes:

- Las expresiones consultadas fueron desarrolladas con base en geometrías específicas, como placas alveolares ([1] y [2]), o secciones aproximadamente rectangulares ([4] y [3]).

- Se realizaron diferentes consideraciones a priori de la longitud de transmisión en el desarrollo de los métodos, la cual difiere en función del empleo del Eurocódigo, el Código Modelo o los Códigos norteamericanos, y de los diferentes modelos de adherencia empleados.

- La influencia de características particulares de la tipología, como la distribución de los tendones o la relación entre el ancho de la losa inferior y de los nervios no son contempladas.

- Los métodos consultados están basados en análisis bidimensionales.

Cabe resaltar, también, algunas reflexiones sobre los resultados del análisis, referentes a la influencia de varios parámetros sobre las tensiones de tracción objeto de estudio.

a) Sobre la longitud de transmisión

La longitud de transmisión es un factor relevante para evaluar las tensiones de tracción producidas durante la transferencia del pretensado. Valorar la longitud de transmisión no es sencillo, dado el gran número de factores que intervienen, y los diferentes criterios existentes, tanto en las normativas y códigos como en la literatura especializada (26) (9).

Se ha comprobado que la relación entre una longitud de transmisión dada y la tensión máxima de tracción por exfoliación obtenida es inversamente proporcional, lo que es coherente con la bibliografía consultada. Así, el cambio de diámetro de los alambres en el primer caso de patología referido, que pasó de $5 \mathrm{~mm}$ a $4 \mathrm{~mm}$ por motivos de producción, redujo la longitud de transmisión, incrementándose el valor de las tensiones de tracción, aunque se mantuviese la fuerza de pretensado total.

\section{b) Sobre la posición del acero y tolerancias constructivas}

Para que se produzcan tensiones de exfoliación, los tendones tienen que estar ubicados fuera del núcleo central de la pieza. El factor $(e-k)$ mide la distancia existente entre la ubicación del tendón pretensado y el borde del núcleo central. Dado que la altura del centro de gravedad en estas piezas se encuentra muy bajo, tratar de centrar la resultante del pretensado incluyendo tendones en la parte superior de los nervios conlleva elevados valores de $(e-k)$ para dichos tendones, y las consiguientes tracciones, a pesar de que la resultante del pretensado total se localice en el entorno del centro de gravedad de la sección.

Además, es interesante comparar las tolerancias constructivas habituales con los valores habituales de $(e-k)$. De acuerdo con la base de datos de placas comerciales consultada, para el pretensado inferior es menor que $2 \mathrm{~cm}$. A su vez, las tolerancias de ejecución fijadas por la Instrucción EHE-o8 son de 5 mm para armaduras activas, y las fijadas por el PCI (27) de 6 $\mathrm{mm}$ para vigas y viguetas, y de $13 \mathrm{~mm}$ para placas alveolares.

c) Análisis de los resultados. Cansancio a tracción

El efecto de la reducción de la resistencia a tracción bajo acciones mantenidas en el tiempo ha sido documentado en el pasado (28) (29).

CM10 (14) recoge una reducción de $f_{c k}$ del 40\% para cargas mantenidas en el tiempo, con base en la referencia (29). No obstante, en el capítulo de diseño, CM10 recomienda un factor de cansancio de valor 1,o para la obtención de las resistencias de cálculo a tracción del hormigón. Indica que la razón de adoptar dicho valor es que el incremento de resistencia en el hormigón después de 28 días compensa la reducción de la resistencia a tracción debido a cargas mantenidas en el tiempo. Señala al respecto que las primeras cargas variables suelen aplicarse meses después de determinar la resistencia a 28 días. Finalmente recomienda aplicar un coeficiente de 0,85 sólo en el caso de que la resistencia se determine a edades superiores a 28 días.

En el caso del Eurocódigo 2 EN 1992-1-1(30), el planteamiento coincide sustancialmente con el de CM9o, y es factible suponer que se sustente en los mismos argumentos.

El problema es que las tracciones provocadas por la transferencia del pretensado en elementos prefabricados se originan en los primeros días, lo cual pone en suspenso la razón seña- 
lada por CM10 para no considerar reducciones de la resistencia a tracción.

Reducciones de resistencia a tracción en el tiempo explicarían la desfavorable evolución de la fisuración descrita en la bibliografía y evidenciada en los casos de patología estudiados, especialmente en el segundo de ellos.

\section{d) Análisis de los resultados. Limitación de la tracción admisible}

El valor límite para las tensiones de tracción calculadas es un aspecto sobre el que no existe acuerdo. CM1o fija $f_{\text {ctd }}$ como límite, mientras CM9o adoptaba para la exfoliación la resistencia a flexotracción minorada por 1,5. Anteriormente, la FIP marcó, para placas alveolares, la resistencia característica a tracción en el momento sin minorar (22). Según la norma EN1168 (23), se debe tomar la resistencia característica a tracción en el momento de la transferencia basada en ensayos. En los tres primeros casos, la ecuación empleada para evaluar las tensiones es la misma [1], y en el último es la [2], con la cual los resultados obtenidos son muy semejantes.

Para obtener un orden de magnitud de la influencia del valor de comparación, al aplicar a la base de datos de placas la ecuación [1], el porcentaje de placas en las que las tracciones calculadas superan los valores de comparación de $f_{\text {ctk.t }}=1,54$ $\mathrm{N} / \mathrm{mm}^{2}$ y $f_{\text {ctm.t }}=2,19 \mathrm{~N} / \mathrm{mm}^{2}$ es de $34 \%$ y $15 \%$ respectivamente, para el pretensado inferior. Si se emplea $f_{\text {ctk,t }} / 1,5$ asciende hasta el 55\%. Tanto en (12) como en (10) se discute el valor a emplear.

\section{CONCLUSIONES Y RECOMENDACIONES}

Los métodos disponibles para la evaluación de las tensiones en la zona de transferencia de piezas prefabricadas con armadura pretesa no convencionales se han mostrado ineficaces, no permitiendo predecir los valores de las tracciones máximas ni explicar los casos de patología documentados.

En este sentido, se destacan los aspectos siguientes:

- La influencia de la configuración geométrica y mecánica de la sección transversal no está correctamente recogida en los métodos consultados. Se han encontrado factores modificadores que consideran estas singularidades.

- La influencia de la ejecución es muy alta, tanto por la elevada influencia de la longitud de transmisión real, como por la estrecha relación entre la posición de los tendones y la magnitud de las tracciones producidas a la luz de las tolerancias constructivas.

- Es importante determinar la longitud de transmisión en la planta de prefabricado, y que sea considerada como parte del criterio de determinación de la edad de transferencia del pretensado. Además, para que estas medidas sean realmente efectivas, se deberían acompañar de la caracterización de la adherencia entre el hormigón y la armadura pretesa en la planta, con el fin de considerar un modelo coherente de evaluación de la tensión. En este sentido, los valores de las tensiones de tracción máximas se incrementan un $40 \%$ al considerar una ley de adherencia lineal en lugar de una uniforme. En los casos de patología estudiados, esta información no se encontraba en las fichas.

- Existen diferencias de criterio significativas a la hora de establecer el valor límite de las tensiones de tracción calculadas. Además, es importante contemplar la evolución en el tiempo de la resistencia a tracción del hormigón por cansancio, que podría reducirse hasta un $40 \%$.

En nuestra opinión, estos aspectos deben ser objeto de revisión y futuras líneas de investigación que avalen métodos que incluyan la influencia relevante de los factores indicados en el margen reglamentario vigente en cuanto a la evaluación de tensiones de tracción en piezas con armadura pretesa adherente.

\section{REFERENCIAS}

(1) Burón, M., Fernández-Ordoñez, D. (1997). Evolución de la prefabricación para la edificación en España. Medio siglo de experiencia. Informes de la Construcción, 48(448): 19-33. http://dx.doi.org/10.3989/ic.1997.v48.i448.963.

(2) Fernández-Ordoñez, D., Fernández, J. (2009). Industrialización para la construcción de viviendas. Viviendas asequibles realizadas con prefabricados de hormigón. Informes de la Construcción, 61(514): 71-79. http://dx.doi.org/10.3989/ ic.09.003.

(3) Gómez, J. et al. (2006). Nuevo edificio terminal. Singularidades, incidencias y soluciones en la ejecución de la obra. Hormigón y Acero, 239: 129-141.

(4) Martínez, M., Sánchez, M. (2011, 25 de octubre). Ejecución de estructuras mixtas, en jácenas de sótanos para aparcamiento de vehículos. Ensayos experimentales sobre su comportamiento mecánico. En $V$ Congreso Internacional de Estructuras de la Asociación Científico Técnica del Hormigón Estructural ACHE (pp. 909-911). Barcelona: ACHE.

(5) Rueda, J. et al. (2014, 3 de junio). Investigación de la fisuración en la zona de transferencia del pretensado de una nueva tipología de piezas prefabricadas para forjados. En VI Congreso Internacional de Estructuras de la Asociación Científico Técnica del Hormigón Estructural ACHE (pp. 33-34). Madrid: ACHE.

(6) Rueda, J., et al. (2014, 3 de septiembre). Transfer zone cracking research of a new type of prestressed floor slab. En 37th International Association for Bridge and Structural Engineering IABSE symposium: Engineering for progress, nature and people (pp. 185-192). Madrid: IABSE. IABSE Symposium Report. International Association for Bridge and Structural Engineering, Vol 102, núm 42, (pp. 185-192).

(7) Rueda, J. (2015). Influencia de la configuración geométrica de placas prefabricadas para forjados en las tensiones originadas por la transferencia del pretensado en elementos realizados con armaduras pretesas ancladas por adherencia (Tesis doctoral no publicada). Madrid: Universidad Politécnica de Madrid.

(8) Ministerio de Fomento (2008). Real Decreto 1247/2008 por el que se aprueba la Instrucción de hormigón estructural (EHE-O8). Centro de Publicaciones de la Secretaría General Técnica del Ministerio de Fomento. 
(9) Vázquez, C. (2000). Estudio comparativo de las propiedades de adherencia de cordones de pretensado en elementos prefabricados de hormigones de altas prestaciones iniciales (Tesis Doctoral no publicada). La Coruña: Universidad de La Coruña.

(10) Uijl, J.A. Den (1991, septiembre). Background of the CEB-FIP Model Code 90 Clauses on Anchorage and Transverse Tensile Actions in the Anchorage Zone of Prestressed Concrete Members. En 28th European Committee for Concrete CEB Plenary Sessions (pp. 71-94). Viena: CEB.

(11) CEB-FIP (1995). Código modelo CEB-FIP 1990 para hormigón estructural, p. 234, Madrid: GEHO-CEB.

(12) Uijl, J.A. Den (1983). Tensile stresses in the transmission zones of hollow-core slabs prestressed with pretensioned strands. En Stevin Laboratory Reports, Vol. 5 of Reports on concrete structures of the Department of Civil Engineering of Delft University of Technology (pp. 1-110). Delft: Delft University of Technology.

(13) CEB (1987). Anchorage zones of prestressed concrete members. Bulletin d'Information n. ${ }^{\circ}$ 181, p. 137. Lausanne: Comité Euro-International du Béton.

(14) CEB-FIP (2013). fib Model Code for concrete structures 2010, p. 348. Lausanne: Ernst \& Sohn.

(15) Dunkman, D.A. (2009). Bursting and spalling in pretensioned U-beams (Tesis Doctoral no publicada). Austin: The University of Texas at Austin.

(16) Gergely, P., Sozen, M.A. (1967). Design of Anchorage Zone Reinforcement in Prestressed Concrete Beams. PCI Journal, 12 (2): 63-75.

(17) Gergely, P., Sozen, M.A., Siess, C.P. (1963). The Effect of Reinforcement on Anchorage Zone Cracks in Prestressed Concrete Members. En Structural Research Series, Vol. 271 of Structural Research Series of University of Illinois (pp. 1-190). Urbana: University of Illinois.

(18) Tuan, C.Y. et al. (2004). End zone reinforcement for pretensioned concrete girders. PCI Journal, 49 (3): 68-82.

(19) Marshal, W.T., Mattock, A.H. (1962). Control of horizontal cracking in the ends of pretensioned prestressed concrete girders. PCI Journal, 7 (10): 56-75.

(20) Arthur, P.D., Ganguli, S. (1965). Test on end-zones stresses in pre-tensioned concrete I beams. Magazine of Concrete Research, 17 (51): 85-96. http://dx.doi.org/10.1680/macr.1965.17.51.85.

(21) French, C. et al. (2011). Cast-in-place concrete conections for precast deck system. En Transportation Research Board of the National Academies, NCHRP 1O-71 Final Report of Transportation Research Board of the National Academies, (1-782). Washington: The National Academies of Sciences, Engineering, and Medicine.

(22) FIP Recommendations (1988). FIP Recommendations: Precast prestressed hollow core floors, p. 4. London: Thomas Telford.

(23) AENOR-CEN (2011). UNE-EN 1168:2006+A3:2011 Productos prefabricados de hormigón. Placas alveolares. Asociación Española de Normalización (AENOR).

(24) ACI 318 (2014). ACI 318-14 Building Code Requirements for Structural Concrete. American Concrete Institute (ACI).

(25) Khonke, P. (1999). ANSYS Theory reference (1999), pp. 14-494. Canonsburg: SAS IP, Inc.

(26) Rueda, J., González, E.(2014). Modelos de transferencia del pretensado: Análisis comparativo. Hormigón y acero, 65 (272): 97-111.

(27) PCI (2006). Tolerance manual for precast and prestressed concrete construction. MNL 135-oo. Third Edition, p. 59. Chicago: Precast / Prestressed Concrete Institute (PCI). USA.

(28) Cook, D.J.,Chindaprasirt, P.(1981). Influence of loading history upon the tensile properties of concrete. Magazine of Concrete Research, 33 (116): 154-16o. http://dx.doi.org/10.1680/macr.1981.33.116.154.

(29) CEB-FIP (2008). Constitutive modelling of high strength/high performance concrete. Fib Bulletin, 42, p. 45. Lausanne: Fédération Internationale du Béton.

(30) CEN (2004). EN 1992-1-1 Eurocode 2: Design of concrete structures - Part 1 - 1: General rules and rules for buildings. European Committee for Standardization (CEN). 